\title{
Agapetes guangxiensis D. Fang (ERICACEAE), A NEW RECORD FOR THE FLORA OF VIETNAM
}

\author{
Nguyen Thi Thanh Huong ${ }^{1 *}$, Tong Yi Hua ${ }^{2,3}$, \\ Vu Tien Chinh ${ }^{1}$, Xia Nian $\mathrm{He}^{2}$, Tran Thi Phuong $\mathrm{Anh}^{4}$ \\ ${ }^{1}$ Institute of Ecology and Biological Resources, VAST, *nthuongiebr@gmail.com \\ ${ }^{2}$ South China Botanical Garden, Chinese Academy of Sciences, Guangzhou 510650, China \\ ${ }^{3}$ University of Chinese Academy of Sciences, Beijing 100049, China \\ ${ }^{4}$ Vietnam National Museum of Nature, VAST
}

\begin{abstract}
Agapetes guangxiensis D. Fang, previously known only from China, is reported here as a new record for the flora of Vietnam. This species was found in Dien Bien and Ha Giang provinces of North Vietnam. It differs from the allied species, A. rubrobracteata, in having leaf blades ovate with serrate margins, inflorescence barcts smaller (c. $0.5 \mathrm{~mm}$ long) and ecalcarate anthers. Information on the taxonomy, type, ecology and distribution is provided for A. guangxiensis. The vouchers are housed at the herbarium of the Institute Ecology and Biological Resources, Ha Noi, Vietnam (HN). A key to the nine species of Agapetes in Vietnam is provided.

Keywords: Agapetes, A. guangxiensis, Ericaceae, New record, Dien Bien, Ha Giang, Vietnam.
\end{abstract}

\section{INTRODUCTION}

Agapetes D. Don ex G. Don with ca. 100 species, belongs to the family Ericaceae under subfamily Vaccinioideae Arn. and tribe Vaccinieae Rchb. [1]. The genus is distributed principally from the East Himalayas through South-West China and Indochina to South-East Asia [2]. Until now Agapetes was considered to be represented in Vietnam by eight species and they were taxonomically studied by several authors [3, 4]. However, during our botanical surveys, an interesting Agapetes species was encountered at $\mathrm{Ha}$ Giang and Dien Bien provinces of North Vietnam. These specimens were characterized by having small ovate leaves with serrate margin, hirsute calyx tube and short white corollas. After comparing the material with collections of Agapetes housed in three southern Chinese herbaria, we determined it as A. guangxiensis D. Fang, a species which hitherto was considered endemic to China [5]. In the paper, we reported here Agapetes guangxiensis as a new record for Vietnam's flora.

\section{MATERIALS AND METHODS}

Specimens of Agapetes at the following herbaria were examined: Institute of Ecology and Biological Resources, Ha Noi (HN); Institute of Tropical Biolgy, Ho Chi Minh City (VNM), VNU University of Science (HNU), National Institute of Medicinal Materials (HNPI), Kunming Botanical Garden (KUN), South China Botanical Garden (SCBG), Guangxi Institute of Sciences (GXMI), Muséum National d'Histoire Naturelle, Paris (P). Specimens of Agapetes guangxiensis collected by the authors in Vietnam are housed in HN.

\section{RESULTS AND DISCUSSION}

\section{Key to the species of Agapetes in Vietnam}

1A. Inflorescences racemose. .2

1B. Inflorescence fasciculate or comprising solitary or paired, axillary flowers

2A. Inflorescences with glandular hairs; bracts large, 6-12 mm long A. bracteata

2B. Inflorescences glabrous; bracts small, 0.5 - $2 \mathrm{~mm}$ long. A. lobbii

3A. Leaf blade longer than $4 \mathrm{~cm}$. .4 


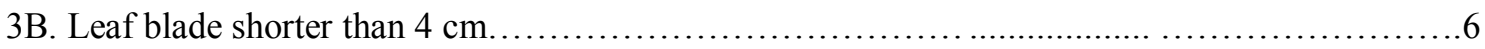

4A. Twigs and calyx densely spreading glandular setose; leaf blade elliptic or oblong-elliptic.......5

4B. Twigs glabrous, calyx velutinous; leaf blade lanceolate A. velutina

5A. Leaf blade 4-6 cm long; corolla $2.5 \mathrm{~cm}$ long A. cauliflora

5B. Leaf blade $6.5-11 \mathrm{~cm}$ long; corolla $2 \mathrm{~cm}$ long A. malipoensis

6A. Leaf margin entire. .7

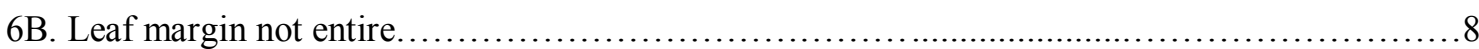

7A. Twigs setose; corolla red.

A. hosseana

7B. Twigs pubescent; corolla white.....

A. mannii

8A. Leaf blade obovate, margin crenulate above middle; bracts large, 1.3-1.5 cm long, anthers calcarate A. rubrobracteata

8B. Leaf blade ovate, margin serrate; bracts small, ca. $0.5 \mathrm{~mm}$ long, anthers ecalcarate. A. guangxiensis

\section{Agapetes guangxiensis D. Fang}

D. Fang in Acta Phytotax. Sin. 36: 276. 1998; R. C. Fang et P. F. Stevens in Fl. China 14: 516. 2005. Type: Guangxi, Napo, Nonghua, limestone hill, in forest, alt. $890 \mathrm{~m}$, Sep. 19th 1983, D. Fang, G. X. Huang \& G. Lin 78397 (holotype \& isotype GXMI!).

Epiphytic shrubs. Twigs terete, ca. $1.5 \mathrm{~mm}$ diam., setose by dense, dark brown, spreading, glandular hairs, glabrescent. Leaves dense; petiole c. 0.8-1.2 mm; leaf blade ovate, 7-12 $\times$ 4-7 mm, coriaceous, glabrous, secondary veins ca. 3 pairs, midvein raised abaxially, impressed adaxially, fine veins inconspicuous, base obtuse, margin slightly revolute, upper parts glandular spiny, apex cuneate, setose. Inflorescences fasciculate, from upper axils, 1-4 flowered. Pedicel c. 3-5 mm, expanded apically, hirsute. Calyx tube turbinate, densely hirsute; limb lobed to base; lobes triangular, c. $1.5 \mathrm{~mm}$, hirsute, glandular pubescent; bracts late ovate, c. $0.5 \mathrm{~mm}$ long. Corolla white, tinged with pale green, subtubular, 9.5-10 mm, slightly 5-angled, angles pubescent; lobes triangular, c. $1 \mathrm{~mm}$, pubescent. Filaments c. $4 \mathrm{~mm}$, pubescent; anthers ca. $3 \mathrm{~mm}$, tubules ca. $2 \times$ as long as thecae, without spurs. Berry globose, $2-6 \mathrm{~mm}$ in diam, setose. Fl. May-Sep, fr. Oct - Dec.

Ecology and habitat: This species is epiphytic or lithophytic in humid evergreen broad-leaved forests at elevations of 1115-1500 m.a.s.l.
Distribution: Vietnam: Dien Bien (Tua Chua), Ha Giang (Quan Ba, Bac Me). China: Guangxi and Yunnan.

Additional specimens examined: Dien Bien, L. Averyanov et al. CPC 1006 (HN); Ha Giang, Q. H. Nguyen et al. CKF071B (HN); HAL $6464(\mathrm{HN})$.

\section{CONCLUSION}

Agapetes guangxiensis species is morphologically the closest ally of A. rubrobracteata but has some striking difference in morphological characteristics such as ovate leaf blade, serrate margin; smaller bracts c. $0.5 \mathrm{~mm}$. long and anthers without spurs. Present report will help prioritizing conservation of this species in Vietnam and China.

Acknowledgment: We are thankful to the curators of the herbaria HNU, VNM, KUN, GXMI, IBSC and PE for their help. The authors also express their thanks to Mrs. Kim Chi for making the illustration.

\section{REFERENCES}

1. Fang D., 1998: Acta Phytotaxonomica Sinica 36(3): 276-278.

2. Fang R. C, Stevens P. F., 2005: Agapetes in Wu C. Y. \& Raven P. R. (eds.), Fl. China 14: 504-517. Science Press, Beijing \& Missouri Botanical Garden Press, St. Louis.

3. Nguyen T. T. H., Nguyen T. H., Tran T. P. A, Vu T. C., Tong Y. H., Xia N. H., 2014: 
Agapetes lobbii C.B. Clarke (Ericaceae), A Newly Recorded Species from Vietnam. Journal of Tropical and Subtropical Botany 22(2): 143-146.

4. Pham Hoang Ho, 1999: An Illustrated Flora of Vietnam, Vol. 1: 623-624 (in Vietnamese). Ho Chi Minh city: Young Publishing House.

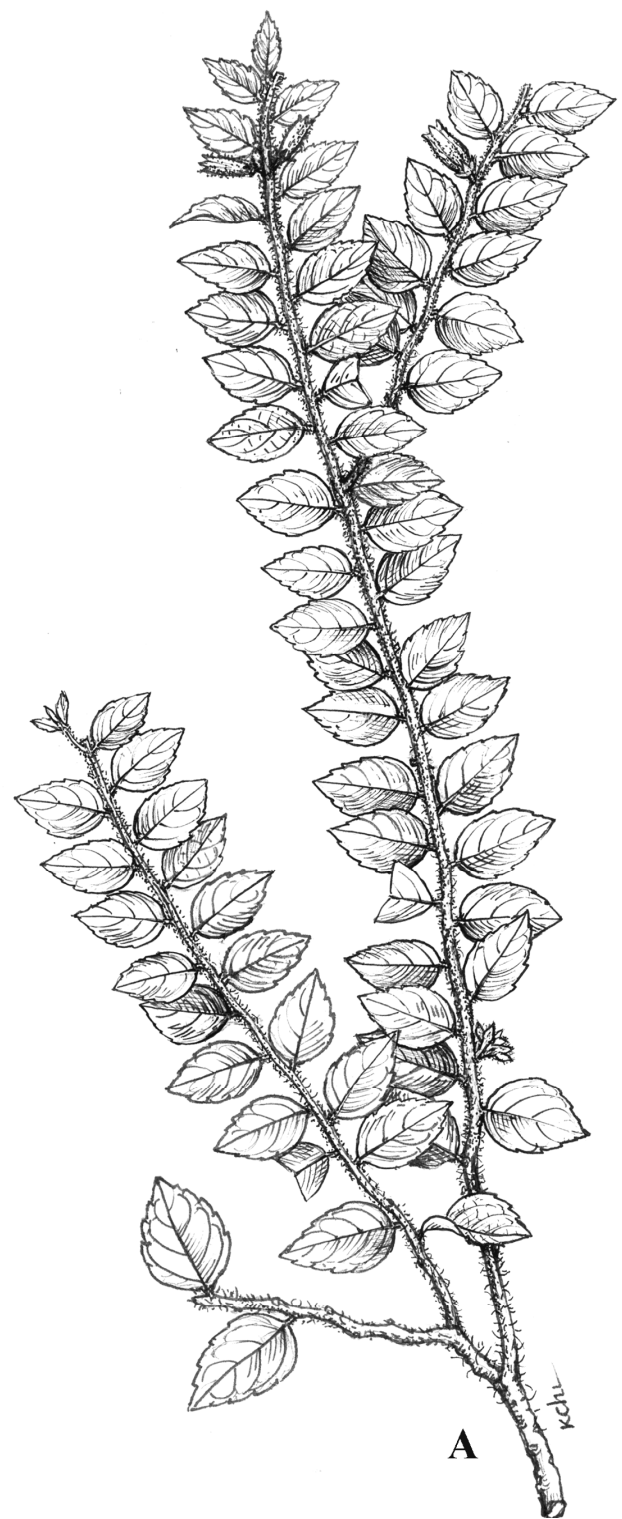

5. Stevens P. F., Luteyn J., Oliver E. G. H., Bell T. L., Brown, E. A., Crowden R. K., George A. S., Jordan G. J., Ladd P., Lemson K., Mclean C. B., Menadue Y., Pate J. S., Stace H. M., Weiller C. M., 2004: Ericaceae in Kubitzki K. (ed.) The Families and Genera of Vascular Plants 6: 145-194. Springer-Verlag, Berlin \& Heidelberg.

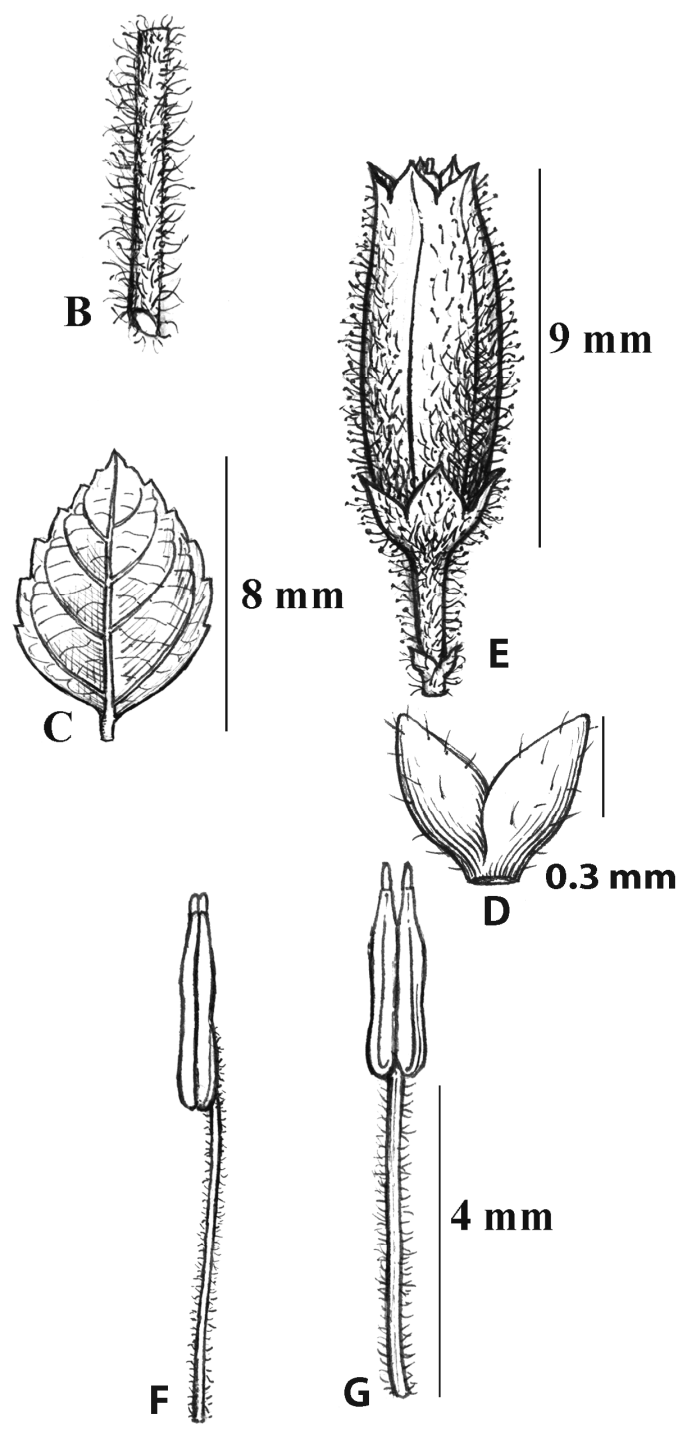

Fig. 1. Agapetes guangxiensis D. Fang

A. Habit; B. Young Branchlets; C. Leaf; D. Bracts;

E. Flower; F, G. Stamens [Drawn by Kim Chi from CPC 1006 (HN)] 


\section{BỔ SUNG LOÀI Agapetes guangxiensis D. Fang (HỌ Ericaceae) CHO HỆ THỰC VẬT VIẸT NAM}

\section{TÓM TẮT}

Trên thế giới, chi Thượng nữ (Agapetes D. Don ex G. Don) thuộc họ Đỗ quyên (Ericaceae Juss.) có khoảng 100 loài, phân bố chủ yếu từ phía Đông dãy Himalaya qua Đông Nam Trung Quốc, Đông Dương sang Đông Nam Á. Ở Việt Nam, chi này có 8 loài, bao gồm cả loài Agapetes lobbi $\mathrm{C}$. B. Clarke phát hiện gần đây. Trong quá trình nghiên cứu chi Thượng nữ (Agapetes) ở Việt Nam, chúng tôi phát hiện loài Agapetes guangxiensis D. Fang có phân bố ở Hà Giang, Điện Biên.

Loài Agapetes guangxiensis có một vài đặc điểm đặc trưng là lá hình trứng nhỏ, mép có răng cưa; ống đài có lớp lông rậm, tràng ngắn, màu trắng. Đây là loài bổ sung cho hệ thực vật Việt Nam và loài này được ghi nhận là đặc hữu của Trung Quốc.

Tù khóa: Ericaceae, Agapetes, Agapetes guangxiensis, ghi nhận mới, Hà Giang, Điện Biên,Việt Nam.

Ngày nhận bài: 22-7-2015 